\title{
A Comparison of Microcompositional Imaging Methods
}

\author{
Michael S. Isaacson
}

Cornell University, College of Engineering, School of Applied and Engineering Physics, W.M. Keck Foundation Program in Nanobiotechnology

241 Carpenter Hall, Ithaca, New York, 14853

More than a half century ago, in a classic paper, Zeitler and Bahr [1] outlined a method for obtaining quantitative information from electron micrographs, thereby extending classical optical methods of microanalysis [2] to even smaller spatial localization scales. In this last decade we have seen an explosion in the quantitative analysis of micro-images. In particular, there has been an almost exponential growth in the coupling of microscopic imaging with spectroscopic methods. This has occurred in all forms of microscopy, from optical, to electron, ion and X-ray microscopy, and more recently to scanned tip imaging microscopies.

Because these various microcharacterization methods (fashionable now to call them nanocharacterization methods) have evolved from many different disciplines using many different tools and techniques, the "local" language used to describe and evaluate the quantitation is not often translatable with ease from one technique to the next. In addition, because there are so many techniques available today which can couple an image with local compositional information, it becomes difficult for the user of a particular method (as opposed to the practitioner of the method) to understand the physical basis of the technique and thus to correlate information from various analytical methods. Moreover, with different "languages" or "currencies", the similarities and complementarities of various methods are not always transparent. In this presentation, I will try to develop a common "language" for microcharacterization so that similarities of the methods become obvious and one can easily look at the advantages and limitations of the various methods.

Of course, in any such discussion, for simplicity, one must make approximations ( and a certain number of disclaimers and caveats) so as to not obscure the basic features of the methods. The aim in this paper is not so much to be all-inclusive, but rather to present a framework for looking at the different techniques.

Therefore, in this presentation we will make the following assumptions: 1) we will concentrate only on electromagnetic radiation "beams" (i.e., photons, electron, ions); 2) we will assume an incoherent beam interrogating the sample so that no beam particle influences the other; 3 ) we will assume a beam of circular cross-section and uniform flux rate (particles/sec/unit area). With these caveats, it can be shown $[3,4]$ that the detected signal rate (counts per second), $\mathrm{S}_{\mathrm{A}}$, emanating from $\mathrm{N}_{\mathrm{A}}$ atoms (or molecules) of type $\mathrm{A}$ in the irradiated volume using a beam of flux rate, J(particles/sec/unit area) is given by:

$$
\mathrm{S}_{\mathrm{A}}=\mathrm{N}_{\mathrm{A}} \mathrm{J} \sigma_{\mathrm{A}} \mathrm{Y}_{\mathrm{A}} \mathrm{F}_{\mathrm{A}}
$$

Where $\sigma_{\mathrm{A}}$ is the cross-section (area) for the primary interaction process, $\mathrm{Y}_{\mathrm{A}}$ is the yield of the secondary interaction process (e.g. if one is detecting the primary process, then $Y_{A}=1$ ). $F_{A}$ is the efficiency of the detection process which includes the efficiency of the detected interaction product 
reaching the detector, the production of the wanted interaction product by other primary and secondary products and the detection efficiency of the detector itself.

This basic microcharacterization equation is general so it is useful in comparing different techniques. This expression, when properly applied, can be used in "standardless analysis" methods found in the literature. For example, the ratio of the number of atoms of type A to those of type B can be obtained from equation (1) as:

$$
\mathrm{N}_{\mathrm{A}} / \mathrm{N}_{\mathrm{B}}=\left[\mathrm{S}_{\mathrm{A}} / \mathrm{S}_{\mathrm{B}}\right] \mathrm{x}\left[\sigma_{\mathrm{B}} \mathrm{Y}_{\mathrm{B}} \mathrm{F}_{\mathrm{B}} / \sigma_{\mathrm{A}} \mathrm{Y}_{\mathrm{A}} \mathrm{F}_{\mathrm{A}}\right]
$$

if the signals were acquired simultaneously. For example, for electron beam X-ray analysis, the quantity in the brackets is just related to the Cliff-Lorimer k factors [5]. For electron beam induced Auger analysis, the quantity in the brackets is just the relative sensitivity[6].

Of course, more exact calculations need to take into account that the signal rate is not simply a product of multiplicative factors. In this presentation we will present examples of the basic microanalysis equation (1) from EDX, EELS, RBS, AES, PIXE, etc. It is the purpose here to tie the various techniques together and provide a correlative framework between them.

References:

[1] E. Zeitler and G.F. Bahr, Exp. Cell Res. 12 (1957) 44.

[2] E.M. Chamot, Elementary Chemical Microscopy, John Wiley and Sons, New York, 1915.

[3] M.S. Isaacson, Micro/Nanocharacterization: The Physics and Methodology of Materials Characterization from Volumes Less Than a Cubic Micron”, Kluwer/Plenum Press, New York (in press).

[4[ M.S. Isaacson, Ultramicroscopy, 49 (1993) 171.

[5] G. Cliff and G.W. Lorimer, J. Microscopy, 103 (1975 )203.

[6] L.E. Davis et.al., Handbook of Auger Electron Spectroscopy, Perkin-Elmer Corp., Physical Electronics Industries, Inc., (1976).

[7] The author would like to thank Dangsheng Su and Gianluigi Botton, who organized this symposium in honor of Elmar Zeitler, a man who has always tried to instill in microscopy researchers the need to be quantitative and not mere"stamp collectors". In addition, I would like to thank Prof. Dr. Zeitler for the many years as a colleague, collaborator, friend and aficionado of jazz. 